NASA/TM-2000-210249

AIAA-2000-2838

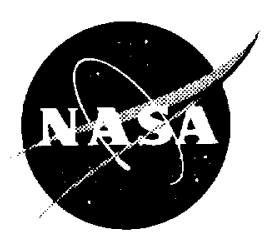

\title{
A 3-D Magnetic Analysis of a Linear Alternator for a Stirling Power System
}

Steven M. Geng and Gene E. Schwarze

Glenn Research Center, Cleveland, Ohio

Janis M. Niedra

Dynacs Engineering Company, Inc., Brook Park, Ohio

Prepared for the

35th Intersociety Energy Conversion Engineering Conference sponsored by the American Institute of Aeronautics and Astronautics

Las Vegas, Nevada, July 24-28, 2000

National Aeronautics and

Space Administration

Glenn Research Center 


\section{Acknowledgments}

The authors wish to acknowledge the Stirling Technology Company for providing hardware information and test data relative to the DOE/STC Technology Demonstration Convertor.

This report contains preliminary

findings, subject to revision as

analysis proceeds.

Trade names or manufacturers' names are used in this report for identification only. This usage does not constitute an official endorsement, either expressed or implied, by the National Aeronautics and Space Administration.

Available from

NASA Center for Aerospace Information 7121 Standard Drive

Hanover, MD 21076

Price Code: A03
National Technical Information Service 5285 Port Royal Road Springfield, VA 22100

Price Code: $\mathrm{A} 03$ 


\title{
A 3-D MAGNETIC ANALYSIS OF A LINEAR ALTERNATOR FOR A STIRLING POWER SYSTEM
}

\author{
Steven M. Geng and Gene E. Schwarze \\ National Aeronautics and Space Administration \\ Glenn Research Center \\ Cleveland, Ohio 44135 \\ Janis M. Niedra \\ Dynacs Engineering Company, Inc. \\ Brook Park, Ohio 44142
}

\begin{abstract}
$\underline{\text { ABSTRACT }}$
The NASA Glenn Research Center and the Department of Energy (DOE) are developing advanced radioisotope Stirling convertors, under contract with Stirling Technology Company (STC), for space applications. Of critical importance to the successful development of the Stirling convertor for space power applications is the development of a lightweight and highly efficient linear alternator.

This paper presents a 3-D finite element method (FEM) approach for evaluating Stirling convertor linear alternators. Preliminary correlations with open-circuit voltage measurements provide an encouraging level of confidence in the model. Spatial plots of magnetic field strength $(\mathrm{H})$ are presented in the region of the exciting permanent magnets. These plots identify regions of high $H$, where at elevated temperature and under electrical load, the potential to alter the magnetic moment of the magnets exists. This implies the need for further testing and analysis.
\end{abstract}

\section{INTRODUCTION}

Early linear alternator designs developed under the NASA Civil Space Technology Initiative (CSTI) generally used rare earth permanent magnets attached to a moving plunger. The harmonic motion of the magnets between the two gaps formed by the inner and outer stator laminations generated a sinusoidal flux in the stator. The sinusoidal flux then induced a voltage in the coils, which were wound inside the outer stator. The magnetic flux path through any of the stator laminations was coplanar with the axis of mover motion; therefore, the designs were somewhat symmetric about their axis of motion. In these early linear alternator designs, a 2-D, finite element method (FEM) analysis was found to adequately model the magnetic fields.

Figure 1 shows a cross-section of the linear alternator designed for the Space Power Demonstrator Engine (SPDE), which was developed in the 1980s by Mechanical Technology, Inc. (MTI) of Latham, New York. Reference 1 provides a discussion on the 2-D analyses that were performed. Reference 2 shows that the analytical open-circuit RMS voltage (i.e., theoretical value) of the SPDE linear alternator was within a few percentage points of the measured values over a wide frequency range.

Current linear alternator designs being developed for the Stirling radioisotope power systems for deep space missions feature stationary rare earth permanent magnets attached to the stator laminations. These designs obtain a sinusoidal, reversing magnetic flux perpendicular to the planes of the stator coils by varying the reluctance of the flux path, as seen by the individual magnets. One coil is wound around each of the stator legs. A sinusoidal voltage is induced in the coils as the moving plunger (or mover) oscillates adjacent to the permanent magnets. These designs are typically symmetric about the axis of motion to the quarter section and, as a result, a 2-D analysis can not accurately model the magnetic fields. In addition, the magnetic flux path is perpendicular to the axis of mover motion, which further complicates the issue. Linear alternators of this design are better modeled in 3-D. 
Figure 2 shows a cross-section of the linear alternator designed for the 55W Technology Demonstration Convertor (TDC), which was developed in the 1990s by Stirling Technology Company (STC) of Kennewick, Washington, for the Department of Energy (DOE). A more detailed description of the TDC is given in References 3 and 4 . The linear alternator model and results discussed in this paper are based on this STC 55W TDC linear alternator design.

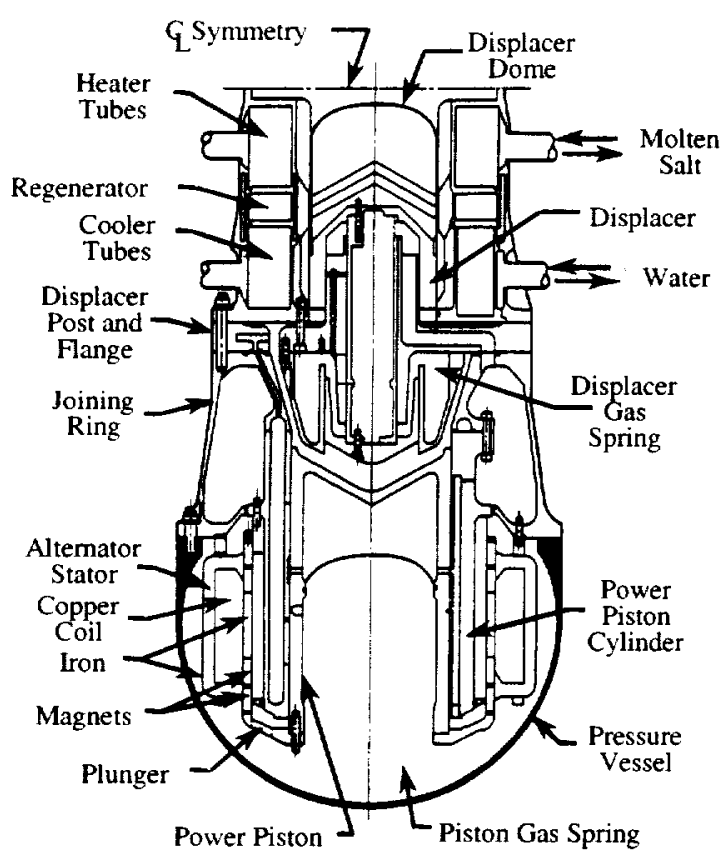

Figure 1 - Cross-section of Stirling Power Demonstrator Engine (SPDE).

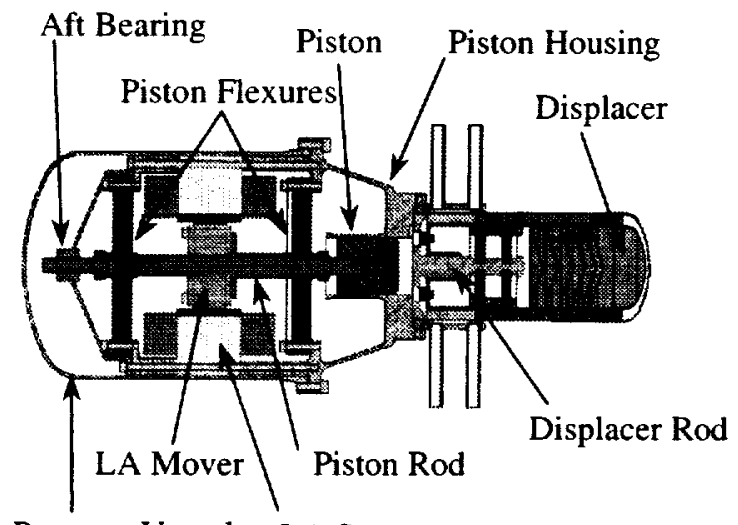

Pressure Vessel LA Stator

Figure 2 - Cross-section of STC's 55W Technology Demonstration Convertor (TDC).

\section{OBJECTIVE}

The objective of this work was to develop a numerical analysis model that could aid engineers in the design, development, evaluation, and understanding of advanced linear alternators.

\section{LINEAR ALTERNATOR MAGNETOSTATIC MODEL}

A three-dimensional magnetostatic model of STC's $55 \mathrm{~W}$ linear alternator was developed using Ansoft's Maxwell 3-D finite element method (FEM) software (Ref. 5). The magnetostatic model consisted of a quarter section of the linear alternator and included the following components: 1) $1 / 4$ stack of Hyperco-50 mover laminations, 2) Neodymium-Iron-Boron halfmagnets, 3) 1/4 stack of Hyperco-50 stator laminations, and 4) copper half-coils. Since the actual hardware is symmetric to the quarter section about the axis of motion, only a quarter section was needed in the model.

Figure 3 shows the flux density (B) and field strength (H) data used in the model for the Hyperco-50 mover and stator laminations. The data shown in this plot was measured at the NASA Glenn Research Center. For illustrative purposes in the FEM model, a remanence $B_{r}$ of 1.26 Tesla and a coercive force $H_{c}$ of $-931 \mathrm{kA} / \mathrm{m}$ were assumed. These values are consistent with the STC specifications for the magnets and are similar to the properties of UGIMAX $37 \mathrm{~B}$ at $23^{\circ} \mathrm{C}$.

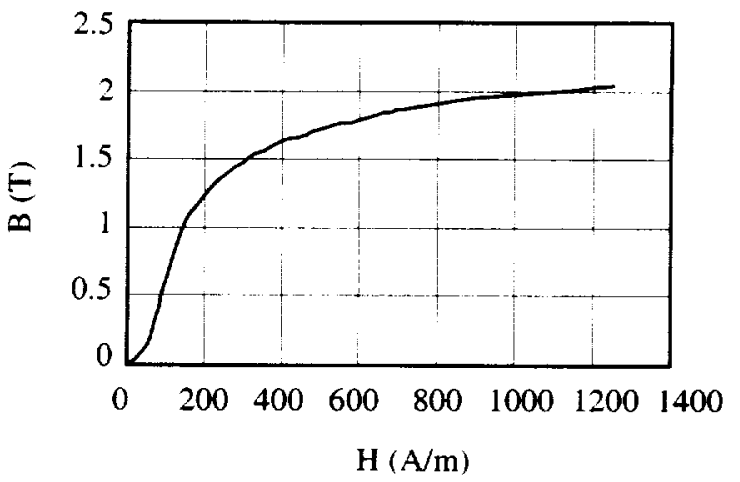

Figure 3 - BH Curve of Hyperco-50.

The primary model parameter of interest for verification with experimental data is the magnetic field parallel to the axes of the coil windings. It is this value of magnetic flux that is used to calculate the opencircuit voltage. To reduce the numerical error associated with the FEM mesh that is used to calculate the magnetic flux, namely the mesh for the coil, stator, 
and coil/stator gap, 9 cut-planes were defined along the axis of the coil. The 9 cut-planes are shown in Figure 4. The model was set-up to calculate and average the magnetic flux normal to each of these cutplanes to obtain an overall average flux parallel to the axis of the coil.

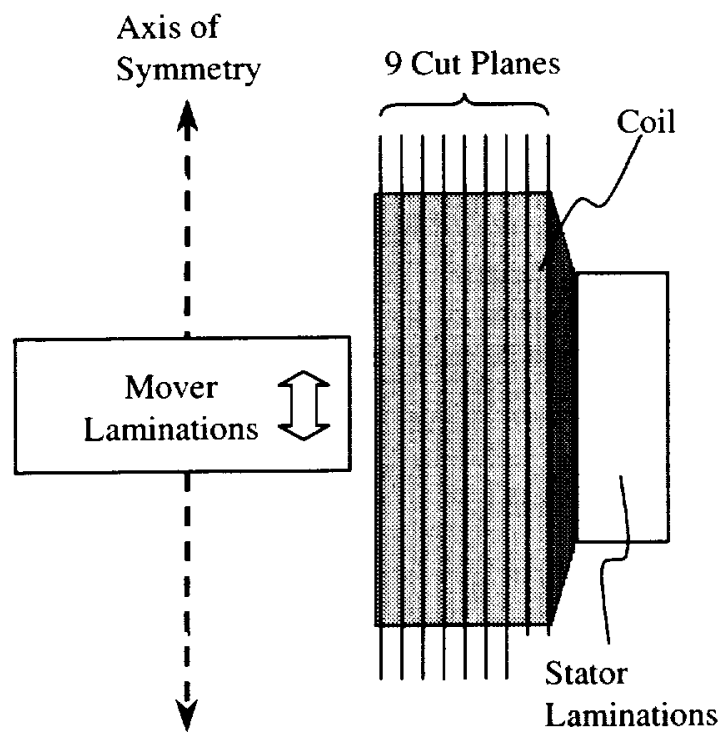

Figure 4 - Cut-Planes Through Coil Used to Calculate Average B Perpendicular to Coil Plane.

Magnetostatic flux solutions for a series of 13 different mover positions were generated. Faraday's Law of induction was then used to calculate the induced electromotive force (emf) in the linear alternator coil windings at the 13 mover positions. The calculated induced emf was then curve-fit, using the TableCurve software. The open-circuit RMS voltage was calculated using the amplitude of the curve-fit solution.

The linear alternator model being a static simulation, eddy current effects due to the mover motion could not be calculated. However, neglecting the eddy current effects should not introduce large errors to the static solutions, since the mover oscillates at a low frequency $(\approx 80 \mathrm{~Hz})$ and all of the magnetic flux conducting structures were laminated (except for the magnets).

\section{$\underline{\text { RESULTS }}$}

\section{Internal State of Magnets}

Figure 5 shows the demagnetization curves estimated for the UGIMAX $37 \mathrm{~B}$ magnets at $23^{\circ} \mathrm{C}$ and $75^{\circ} \mathrm{C}$. The $23^{\circ} \mathrm{C}$ demagnetization curve was used in the magnetostatic analysis, since STC measures the linear alternator open-circuit voltage with the magnets at room temperature. Several load lines are shown in this figure with respect to one particular magnet. The load lines representing the mover positioned adjacent to this magnet and away from this magnet (i.e. at the ends of the mover stroke) were calculated based on the magnetic flux density $B$ and magnetic field strength $\mathrm{H}$ averaged over the volume of the magnet. For the case where the mover is positioned adjacent to the magnet, the average load line is a fairly good representation of the internal operation of the magnet, since $B$ and $H$ are then somewhat uniform. But for the case where the mover is positioned away from the magnet, large variations in $H$ exist. The average load line in this case may indicate a false margin of safety relative to demagnetization. Load lines for small, localized areas of the magnet may approach the critical knee where demagnetization can occur.

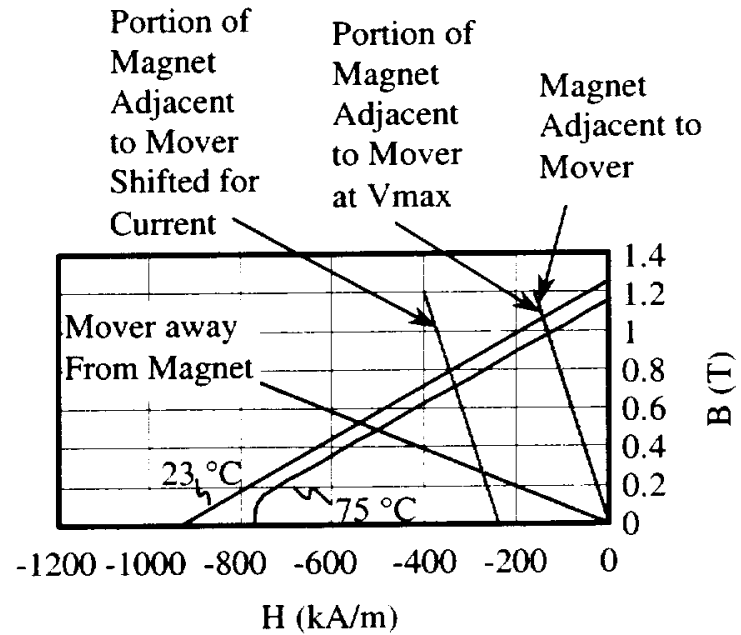

Figure 5 - Demagnetization Curve for UGIMAX 37B NeFeB Permanent Magnets along with Load Lines for the 55W TDC Linear Alternator.

Figure 5 also shows a load line for one of the magnets at the mid-point of the mover stroke, which corresponds with the maximum induced voltage in the coils. In this case, approximately half of the magnet is positioned adjacent to the mover. This load line was calculated based on the average $\mathrm{B}$ and $\mathrm{H}$ for only the portion of the magnet positioned adjacent to the mover. The reason for averaging over only this portion is that it is only this portion that is within the magnetic circuit at the mover mid-stroke position. This load line practically falls right on top of the magnet-adjacent-tomover load line described earlier. The load line was then shifted by $-238 \mathrm{kA} / \mathrm{m}$ (number of turns multiplied by the peak current divided by the thickness of the 
magnet and air gap) to account for the demagnetization effect caused by current flowing through the coil. Equations derived by Niedra (Ref. 6, Appendix A) were used to verify the load lines for the covered portions of the magnet and agreed well with the FEM model. Based on the load lines shown in Figure 5, it appears that the self-demagnetization effect of the uncovered magnets are more of a concern than the demagnetization effect caused by current flowing through the coils. A closer look at localized areas of the magnets is needed.

Figure 6 is a plot of magnetic field strength $(\mathrm{H})$ vs. distance along one stator leg for the mover positioned at mid-stroke. This is the mover position for peak induced voltage in the coils. The plot shows three curves plotted along parallel lines which cut through the magnets: one represents a line along the inside surface (mover side of magnet), one represents a line along the center, and one represents a line along the outside surface (stator side of magnet) of the magnet pair. Each line is parallel to the mover motion (the $z$-direction) and lies in the plane of the magnet symmetry. This plot shows a localized maximum demagnetization field of roughly $670 \mathrm{kA} / \mathrm{m}$ on the inside surface of the uncovered magnets. The plot also shows a field spike of $680 \mathrm{kA} / \mathrm{m}$ on the inside surface of the magnets, where the two magnets meet. This spike is due to $\mathrm{H}_{z}$, (the $\mathrm{H}$ in the $\mathrm{z}$-direction), as predicted by Ampere's Law. The curves for the center and outside magnet surfaces in the covered regions of the magnets show a much smaller variation in the demagnetization fields. A further refinement of the FEM mesh in this region of the model should be explored to resolve irregularities of computation.

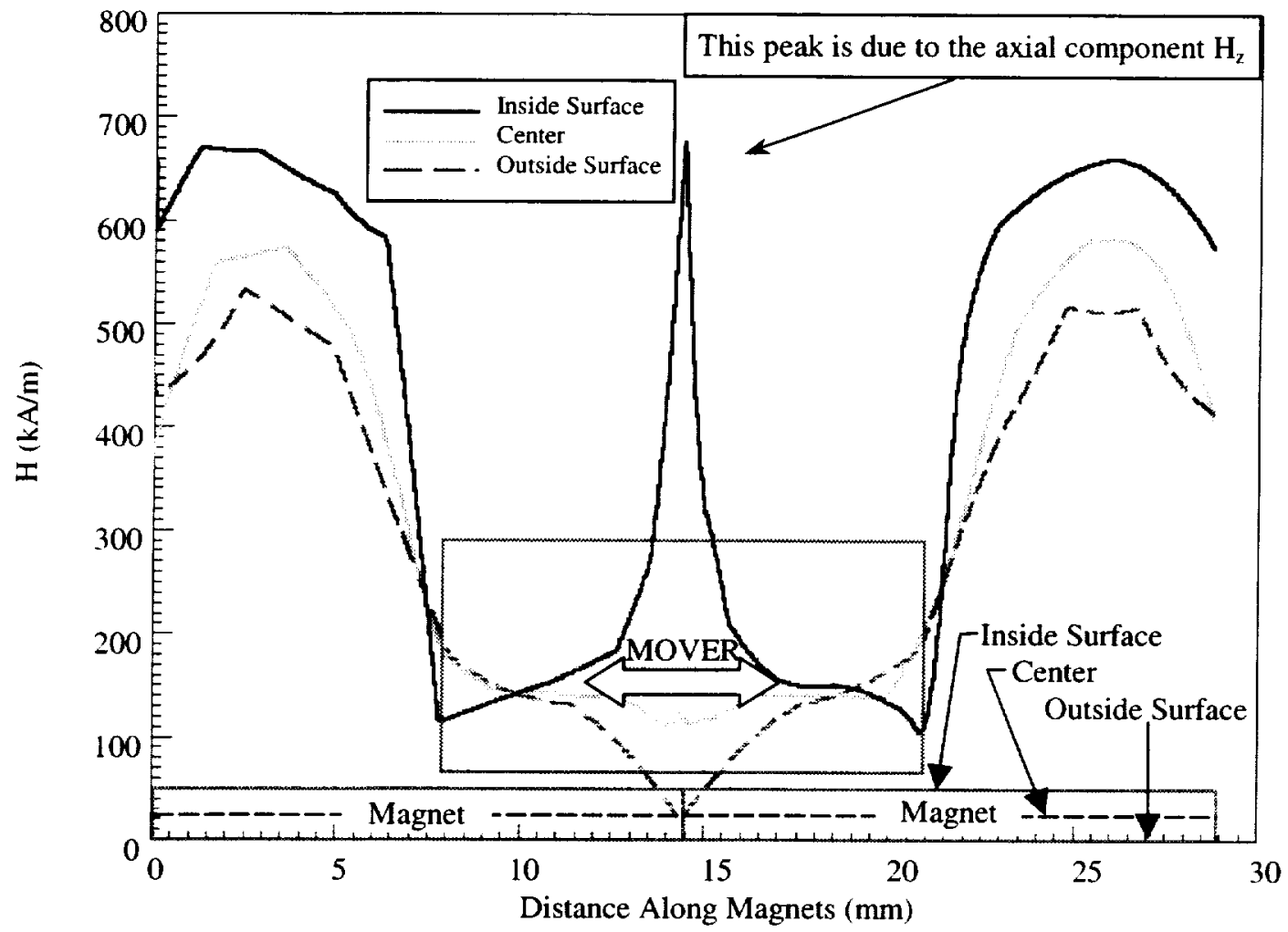

Figure 6 - Magnetic Field Strength $|\overline{\mathrm{H}}|$ vs. Distance along Magnets at $23^{\circ} \mathrm{C}$. 
Average Properties at a Higher Temperature

In actual Stirling convertor applications, the magnet temperatures may reach as high as $75^{\circ} \mathrm{C}$. The model was used to generate the $\mathrm{BH}$ trace for the magnets over a cycle at the expected operating temperature. Figure 7 shows a plot of average $B$ vs. average $H$, for the alternator magnets as the mover oscillates with a 6-mm amplitude. $B$ and $H$ are averaged over the volume of the magnet. This plot shows that the minimum average values of $B$ and $H$ are 0.58 Tesla and $-503 \mathrm{kA} / \mathrm{m}$, respectively. The critical $\mathrm{H}$ value for the NeodymiumIron-Boron magnets assumed in the model is approximately $-750 \mathrm{kA} / \mathrm{m}$ at $75^{\circ} \mathrm{C}$. For $\mathrm{H}$ values $\leq-750 \mathrm{kA} / \mathrm{m}$, demagnetization may occur. Based on the large variations in the magnetic field strength seen in the magnets at the lower temperature, peak $\mathrm{H}$ values for localized portions of the magnets may exceed -750 $\mathrm{kA} / \mathrm{m}$ at the higher temperature.

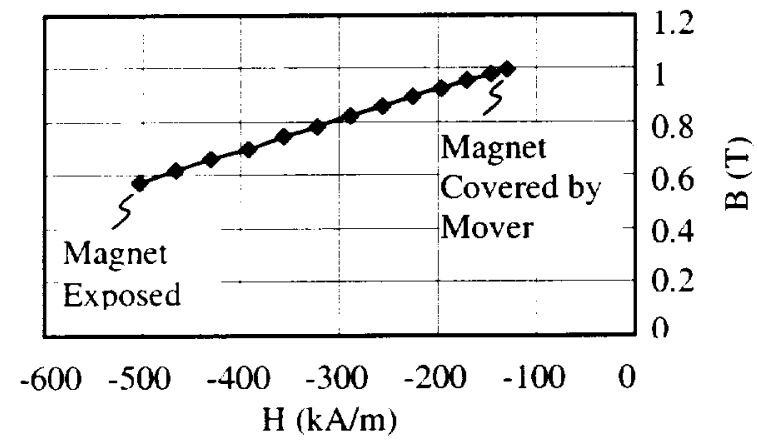

Figure 7 - Magnetic Flux Density vs. Field Strength Averaged over Magnet Volume at $75^{\circ} \mathrm{C}$.

Induced Voltage

Figure 8 shows a plot of the magnetic flux linking each alternator coil as a function of mover position. Although the peak flux of $1.52 \mathrm{E}-4$ Webers occurs at the ends of the mover stroke, the largest change in magnetic flux with respect to time (and therefore the largest induced emf in the coils) occurs at the mid-point of the mover stroke. The plot shows that the flux is almost linear with respect to mover position. The fact that this plot is not perfectly linear may be due to minor variations in flux fringing with mover position or it may indicate minor inaccuracies associated with the numerical solution.

The magnetostatic flux solutions were considered converged, once the energy error between iterations (as defined by the Maxwell software) became less than 2\%. The $2 \%$ convergence criterion was selected as a tradeoff between accuracy and computation time.
Figure 9 shows a plot of the predicted alternator opencircuit voltage as a function of time. The diamond markers show the model predictions. The solid line is a sine-wave curve fit of the points. The curve fit was used to calculate the RMS open-circuit voltage for comparison with the measured value. The shape of the voltage curve should be nearly sinusoidal. The fact that the model predictions do not lie on a sinusoidal curve may once again be due to either flux fringing variations or numerical inaccuracies (c.f. Fig. 8).

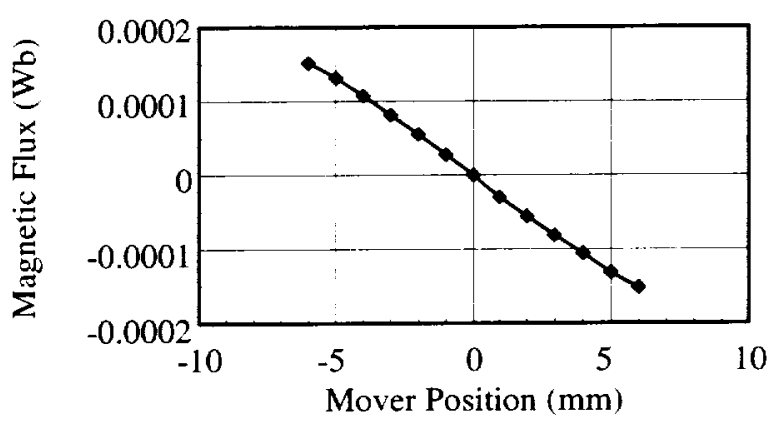

Figure 8 - Magnetic Flux vs. Mover Position at $23^{\circ} \mathrm{C}$.

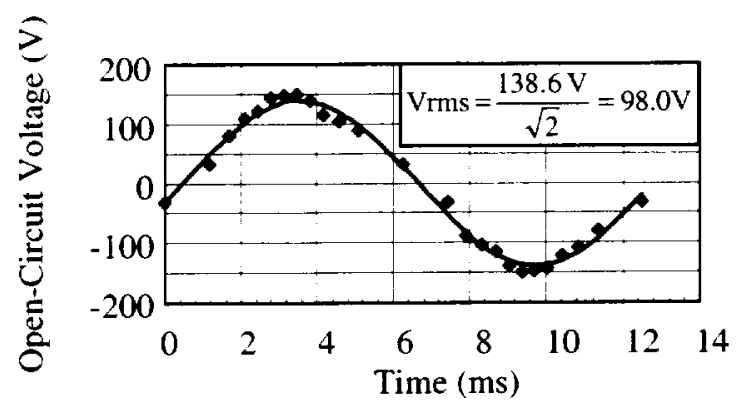

Figure 9 - Open-Circuit Voltage vs. Time at $23^{\circ} \mathrm{C}$.

As with the earlier SPDE open-circuit voltage comparisons to 2-D analytical predictions, the TDC open-circuit voltage correlates closely with the new preliminary 3-D analysis. As the model is developed further and validated with more comprehensive test data, it will be an increasingly valuable design tool.

\section{CONCLUSIONS}

A commercial magnetics computation tool has been applied to develop a valuable numerical analysis model of linear alternator designs for Stirling power convertors. This model can be used to explore design variations early in the development process. However, the model needs to be further validated through additional experimentation. 
The results shown by the preliminary 3-D analysis indicate that a more comprehensive analysis and measurements are appropriate for the 55W TDC linear alternator design, especially since the magnet temperatures may reach $75^{\circ} \mathrm{C}$ in space power applications. The portion of the magnet with the highest potential for demagnetization appears to be on the surface of the magnet pair at the edge, where the magnets are in contact with each other and adjacent to the mover. The region near an advancing/receding edge of the mover is a location where fringing fields due to load current can reinforce the already high selfdemagnetizing field in the area of a magnet not adjacent to the mover. The results presented here support the need for a detailed study of load current effects in these critical regions.

Special care is needed when selecting magnets because of the high, localized demagnetizing fields. Magnets with a higher resistance to demagnetization are being used in the TDC convertors currently being fabricated by STC for NASA Glenn.

\section{REFERENCES}

1. Brown, A.T.: "Space Power Demonstrator Engine, Phase I Final Report," NASA CR-179555, 1987.

2. Dochat, G.: "SPDE/SPRE Final Summary Report," NASA CR-187086, 1993.

3. Thieme, L.G., Qiu, S., White, M.A.: "Technology Development for a Stirling Radioisotope Power System," NASA TM-2000-209791, 2000.

4. White, M.A., Qiu, S., Augenblick, J.E.: "Preliminary Test Results from a Free-Piston Stirling Engine Technology Demonstration Program to Support Advanced Radioisotope Space Power Applications," in Proceedings from STAIF-00, Space Technology and Applications International Forum-2000, Albuquerque, New Mexico, 2000.

5. Ansoft's Maxwell 3-D simulator, see World Wide Web page: www.ansoft.com

6. Niedra, J.M.: "Lightweight Linear Alternators With and Without Capacitive Tuning," NASA CR-185273, 1993. 

